\title{
Profilaxia da trombose venosa profunda em pós-operatório de cirurgias ortopédicas em um hospital de traumato-ortopedia
}

\author{
Prophylaxis of deep venous thrombosis in the posthoperative period of orthopedic surgeries in a \\ traumato-orthopaedic hospital
}

Marcio A. Barros-Sena ${ }^{1}$

Marcelo Genestra ${ }^{2}$

\begin{abstract}
O tromboembolismo venoso é uma patologia potencialmente fatal que pode complicar a recuperação de pacientes hospitalizados. Pacientes especialmente submetidos a procedimentos cirúrgicos de grande porte estão sob risco. A trombose venosa profunda (TVP) e a embolia pulmonar (EP) são complicações pós-operatórias reconhecidamente difíceis de diagnosticar, e o tratamento pode, freqüentemente, ser iniciado demasiadamente tarde para ser eficaz. Sem profilaxia, um em cada quatro pacientes com idade superior a 40 anos, submetidos a cirurgia geral de porte com mais de uma hora de duração, desenvolverão trombose venosa profunda. Os pacientes de risco podem ser identificados e receber profilaxia - um método menos oneroso para reduzir a morbidade e a mortalidade. Desta forma, a prática de Atenção Farmacêutica, realizada em pósoperatório de cirurgia ortopédica constitui-se numa ferramenta útil para evitar a progressão desta patologia. Através da identificação dos sintomas sugestivos consegue-se prevenir o agravamento desta doença, o que torna mais fácil a sua cura. Rev. bras. hematol. hemoter. 2008;30(1):29-35.
\end{abstract}

Palavras-chave: Trombose venosa profunda; atenção farmacêutica; heparina de baixo peso molecular.

\section{Introdução}

Pouco conhecida pela população, porém responsável por milhares de mortes todos os anos, a trombose venosa profunda (TVP) é uma doença complexa e silenciosa que pode trazer sérias complicações quando não tratada de forma rápida e adequada. Ela consiste no desenvolvimento de um coágulo - também chamado de trombo, dentro de um vaso sangüíneo venoso com conseqüente reação inflamatória do vaso, podendo, esse trombo, determinar obstrução venosa total ou parcial, levando à interrupção do fluxo sangüíneo. A complicação aguda desta doença, que é séria e pode ser mortal, é a embolia pulmonar (EP). ${ }^{1}$
A complicação crônica que surge dois a cinco anos após a TVP é chamada síndrome pós-flebítica (insuficiência venosa crônica), que tem um grande impacto sobre a qualidade de vida e sobre os custos da assistência médica. Acontece porque, em boa parte dos pacientes, as veias nunca mais se desobstruem ou ficam danificadas com a alteração em suas paredes e válvulas, caracteriza-se pela formação de úlceras nas pernas, inchaço e dor vespertina, devido às alterações e cicatrizes deixadas pela TVP no sistema venoso. Esta patologia tem uma grande importância socioeconômica, já que inúmeros trabalhadores ficam afastados de suas atividades em conseqüência das complicações da doença. As úlceras venosas se desenvolvem em pelo menos 300/100.000

${ }^{1}$ Farmacêutico. Especialista em Análises Clínicas. Ex-estagiário do Instituto Nacional de Traumato-Ortopedia/Ministério da Saúde. ${ }^{2}$ Especialista, Mestre, Doutor e pós-Doutor em Bioquímica. Instituto Oswaldo Cruz-Fiocruz/RJ. Curso de Medicina e Mestrado em Ensino de Ciências da Saúde e Meio Ambiente - UniFOA.

Correspondência: Marcelo Genestra

UniFOA - Centro Universitário de Volta Redonda

Curso de Medicina e Núcleo de Pesquisa - Campus Universitário Olézio Galotti

Av. Paulo Erlei Alves Abrantes, 1325 - Três Poços

27240-560 - Volta Redonda-RJ - Brasil

Tel.: (24)3346-8400 - Fax.: (24)3340-8404

E-mail: marcelo.genestra@foa.org.br 
habitantes, e a proporção devida à TVP é de aproximadamente $25 \%$. Estimou-se que o custo anual de tratamento das úlceras venosas seria de 400 milhões de libras para o Reino Unido e de mais de 1 bilhão de dólares para os Estados Unidos. A ocorrência da síndrome pós-flebítica e de suas sérias complicações reforça a necessidade da realização da profilaxia do TEV em pacientes submetidos a procedimentos cirúrgicos. ${ }^{30}$

A trombose venosa é uma doença que nos últimos anos assumiu grande importância, apesar de serem conhecidas suas complicações há vários anos, visto que a embolia pulmonar sempre foi temida; entretanto, não se tinha idéia clara de sua importância na mortalidade e morbidade associadas a outras doenças. ${ }^{3}$

Desde o começo do século XX se fala em prevenir o tromboembolismo venoso em pacientes acamados ou cirúrgicos, porém só nos últimos 25 anos é que houve uma preocupação generalizada com esta questão e um aprofundamento nas pesquisas de métodos profiláticos. ${ }^{10}$ Este fato se deve ao aumento da expectativa de vida e, conseqüentemente, dos fenômenos tromboembólicos envolvidos em procedimentos cirúrgicos cada vez mais complexos. Também se deve ao desenvolvimento de métodos mais sensíveis e objetivos de diagnósticos da TVP, como fibrinogênio marcado com Iodo125 e a flebografia. Estes métodos diagnósticos permitiram também o estudo da profilaxia, e, embora ainda não ideais, são bastante eficientes e seguros, podendo ser aplicados de rotina aos pacientes com risco aumentado de tromboembolismo venoso. ${ }^{27}$

A gênese dos fenômenos tromboembólicos está relacionada com a tríade de Virchow (alterações da parede vascular, alterações do fluxo sangüíneo e alterações de constituintes do sangue). Modernamente sabe-se que estes fatores podem exercer diferentes graus de influência, podendo atuar isoladamente ou em associação com um dos outros dois fatores.

A incidência de TVP e EP no Brasil é alta, ${ }^{25}$ e o tromboembolismo é bastante comum: Castro-Silva estudou $28 \mathrm{mil}$ internações por ano pelo Sistema Único de Saúde (SUS) em conseqüência do tromboembolismo venoso e observou 4.247 (15,71\%) óbitos entre estes doentes. Um outro estudo realizado por Castro-Silva ${ }^{9}$ sobre a prevalência de TEV em Minas Gerais e sua projeção para o Brasil constatou ser essa patologia a quinta causa de hospitalização por doenças cardiovasculares no estado, só superada por aquelas de evolução crônica. A avaliação envolveu mais de dois milhões de internações em 680 hospitais públicos e conveniados do SUS.

A TVP é considerada uma doença multifatorial em que fatores genéticos interagem entre si com fatores ambientais, levando ao desenvolvimento da trombose..$^{24-28,33}$ Isto explica o fato de ocorrer trombose venosa profunda em pacientes aparentemente sem nenhum fator de risco evidente. A cada dia novos fatores genéticos de risco para desenvolvimento de TVP são descobertos com o auxílio das técnicas de biologia molecular.
A profilaxia da TVP está relacionada com a categoria de risco em que o paciente se encontra:

- Pacientes com baixo risco - neste grupo não há evidência para se realizar profilaxia, recomenda-se apenas cuidados gerais ${ }^{10}$;

- Pacientes com risco moderado - neste grupo recomendam-se cuidados gerais mais medidas medicamentosas como heparina não fracionada em baixa dose ou heparinas de baixo peso molecular;

- Pacientes com alto risco - neste grupo recomendamse cuidados gerais mais medidas medicamentosas como heparina não fracionada ou heparina de baixo peso molecular. ${ }^{14}$

As heparinas de baixo peso molecular possuem maior capacidade em inibir o fator Xa e menor afinidade por proteínas plasmáticas, vasculares, células endoteliais, macrófagos e plaquetas, conferindo maior biodisponibilidade e meia-vida plasmática e redução dos efeitos colaterais relacionados a plaquetopenia e risco de sangramento, ${ }^{3,5}$ proporcionam uma economia de custos provocada pela alta hospitalar precoce e favorece o tratamento domiciliar. Estas características são vantagens que as heparinas de baixo peso molecular possuem quando comparadas às heparinas não fracionadas, têm a desvantagem do alto custo, todavia em termos de eficácia e segurança ambas são similares. ${ }^{35}$

As heparinas de baixo peso molecular disponíveis no mercado brasileiro são: nadroparina ou Fraxiparina ${ }^{\circledR}$, da Sanofi-Synthelabo; enoxaparina ou Clexane ${ }^{\circledR}$, da Aventis Pharma Ltda.; e dalteparina ou Fragmin ${ }^{\circledR}$, da Pharmacia (Tabelas 1 e 2).

As contra-indicações ao uso de HBPM são as seguintes: hipersensibilidade ao produto; distúrbios hemorrágicos; úlcera gastroduodenal ativa; interação medicamentosa; endocardite bacteriana; trombocitopenia; e acidente vascular cerebral recente.

Até a primeira metade do século XX, o farmacêutico tinha como função manipular medicamentos e orientar os

Tabela 1. Doses profiláticas das heparinas de baixo peso molecular (Adaptado de Daves \& Faulds)

\begin{tabular}{|c|c|c|c|}
\hline Droga & $\begin{array}{c}\text { Nome } \\
\text { comercial }\end{array}$ & Dose menor & Dose maior \\
\hline Nadroparina & Fraxiparina & $0,3 \mathrm{ml}$ (2.850Ul) & $0,6 \mathrm{ml}$ (5.700Ul) \\
\hline Enoxaparina & Clexane & $20 \mathrm{mg}$ (2.000UI) & $40 \mathrm{mg}$ (4.000Ul) \\
\hline Dalteparina & Fragmin & $2.500 \mathrm{UI}$ & $5.000 \mathrm{UI}$ \\
\hline
\end{tabular}

Tabela 2. Heparina de baixo peso molecular na profilaxia da trombose (Adaptado de Davies \& Faulds, 1996)

\begin{tabular}{ccc}
\hline Condição & $\begin{array}{c}\text { Incidência com } \\
\text { profilaxia }\end{array}$ & $\begin{array}{c}\text { Incidência sem } \\
\text { profilaxia }\end{array}$ \\
\hline Cirurgia geral & $7 \%$ & $25 \%$ \\
Cirurgia de quadril & $15 \%$ & $51 \%$ \\
Cirurgia de joelho & $31 \%$ & $61 \%$ \\
\hline
\end{tabular}


pacientes sobre o seu uso. ${ }^{18}$ Após a década de 50, a industrialização dos medicamentos tomou parte dessa atividade. Como os medicamentos produzidos já vinham com bula, a partir da década de 60 as pessoas passaram a se automedicar. Para romper essa "herança", iniciou-se nos EUA, nos anos 90, o movimento "Atenção Farmacêutica", com a finalidade de resgatar a função do farmacêutico. ${ }^{15}$

A atenção farmacêutica é um modelo de prática desenvolvido no contexto da assistência farmacêutica, na perspectiva da integralidade das ações de saúde; embora pareçam semelhantes, possuem conceitos distintos. A atenção farmacêutica refere-se a atividades específicas do farmacêutico no âmbito da atenção à saúde, enquanto a assistência farmacêutica envolve um conjunto mais amplo de ações, com características multiprofissionais. Estas ações envolvem um ciclo de atividades que vai desde a seleção de medicamentos até a dispensação. ${ }^{11}$

A filosofia da atenção farmacêutica corresponde a uma troca mutuamente benéfica, na qual o paciente confere autoridade ao farmacêutico e este, por sua vez, oferece ao paciente conhecimento e comprometimento com a melhora da sua qualidade de vida. ${ }^{20} \mathrm{~A}$ atenção farmacêutica é elemento essencial aos serviços de saúde e deve estar integrada com as outras partes do sistema e demais profissionais. ${ }^{29,34}$

Entretanto, deve-se salientar-se que um programa de atenção farmacêutica deve estar respaldado por um bom sistema de distribuição de medicamentos, para que os profissionais estejam livres para assumir atividades que envolvem maior proximidade do paciente, o que muitas vezes não é possível, devido à falta de recursos financeiros do paciente para prover o fármaco ou à escassez de recursos dos hospitais públicos para fornecê-los, constituindo desta forma um problema relacionado com o medicamento.

Inserido neste contexto, deve haver um programa de atenção farmacêutica junto ao paciente que desenvolve TVP. $\mathrm{O}$ farmacêutico deve trabalhar junto ao paciente de maneira que este venha a aderir ao tratamento prescrito pelo médico. Muitas vezes o doente tem medo, ou ainda se sente fraco e prefere ficar na cama, apesar da ordem do médico para levantar. Uma das formas de favorecer a aderência ao tratamento é informá-lo sobre as conseqüências de uma TVP para sua saúde, entregando-lhe um folheto explicativo nos mesmos moldes do que é entregue no programa de prevenção realizado no Instituto Nacional de Traumato-ortopedia; desta forma, mesmo que o paciente seja analfabeto, algum membro da família ou um vizinho pode ler e sensibilizar o doente da necessidade de realizar o tratamento.

A orientação farmacêutica não se restringe apenas ao paciente; a família também deve estar ciente da situação e, desta maneira, cooperar para que o paciente venha a aderir ao tratamento, não deixando faltar às consultas para o controle dos anticoagulantes e não permitindo a tomada de qualquer medicamento que, eventualmente, em associação com anticoagulante, possa causar hemorragias (como o ácido acetil salicilico/aspirina), e todos os demais antiinflamatórios, que podem chegar a ser mortais..$^{24,27}$

A interação paciente-família é muito importante, tornando-se uma ferramenta muito útil para a prática da atenção farmacêutica. Esta interação, provocada pelo farmacêutico, além de cooperar para a cura do paciente permite a expansão do conhecimento da doença e, desta maneira, outros pacientes que por algum motivo se encontrarem com TVP e não souberem como proceder poderão receber auxílio deste membro da família ou do próprio paciente curado, e desta forma alcançar a sua cura. $\mathrm{O}$ farmacêutico, através deste trabalho, está promovendo a saúde, sendo bastante importante, pois nem todos os pacientes têm acesso às informações necessárias a respeito da sua patologia e do medicamento de que está fazendo uso; isto pode vir a acarretar cura mais tardia ou desenvolvimento de outra patologia, trazendo como conseqüência elevados gastos financeiros e, em alguns casos, o óbito.

Existem na literatura índices significativos de TVP em pacientes de diferentes especialidades, incluindo clínica médica, ortopedia, cirurgia geral, ginecologia, urologia e cirurgia vascular. Todavia, o objetivo deste trabalho foi analisar a importância da atenção farmacêutica na profilaxia desta patologia em pacientes de pós-operatório de cirurgia ortopédica do Instituto Nacional de Traumato-Ortopedia (Ministério da Saúde), situado na cidade do Rio de Janeiro.

\section{Casuística e Método}

O estudo consistiu numa abordagem retrospectiva quali-quantitativa utilizando dados específicos de registro que constam na Ficha de Profilaxia da Trombose Venosa Profunda no Instituto Nacional de Traumato-Ortopedia/Into de todas as internações que ocorreram no período de agosto a novembro de 2003. Esta ficha hospitalar (questionário fechado), obrigatória em todos os prontuários de pacientes com idade igual ou superior a 12 anos, foi desenvolvida para ser utilizada pelo farmacêutico no momento da alta hospitalar. O levantamento levou em consideração pacientes de ambos os sexos, internados para cirurgia ortopédica de membros inferiores. Levando-se em conta a existência na literatura de altos índices de trombose venosa profunda em pacientes do pós-operatório ortopédico, os critérios de inclusão levaram em consideração a obtenção de permissão da chefia imediata do setor para a realização deste levantamento e a não identificação dos pacientes. Os critérios de exclusão incluíram pacientes externos, não internados e/ou não operados no Instituto Nacional de Traumato-Ortopedia.

Os dados do questionário aplicado a cada paciente permitiram que se identificassem os pacientes que apresentaram sintomas e sinais sugestivos de trombose venosa profunda. Este questionário foi aplicado no momento da alta do paciente, visto que é nesta hora que o medicamento 
enoxaparina (heparina de baixo peso molecular) é dispensado ao paciente, que completa o tratamento profilático em casa. O primeiro contato com o paciente, ainda no leito, consistiu na identificação de fatores de risco e obtenção de outros dados (telefone, idade, medicamentos de alta, etc). Também ainda no leito ocorreu a entrega de um folheto ilustrativo explicando o que é trombose e quais os sinais e sintomas relacionados a esta patologia. O paciente ainda assinava um termo de recebimento das seringas e era orientado a retornar com as mesmas vazias após o uso. Este procedimento tem a finalidade de evitar que o paciente venda o medicamento, além de proteger o serviço de farmácia no caso de auditoria .

Após a alta, foram realizados três contatos telefônicos com cada paciente: o primeiro, feito uma semana após a alta, onde se indagou se o paciente aderiu ou não ao tratamento e se sinais e sintomas sugestivos de TVP se fizeram presentes. O segundo contato foi feito um mês após a alta. O terceiro contato foi realizado dois meses após. Caso algum sinal/sintoma sugestivo de TVP se fizesse presente, o paciente era orientado a retornar ao hospital imediatamente para tratamento imediato.

A fim de definir a estratificação de risco para trombose venosa profunda para cada paciente foram pesquisados fatores clínicos, medicamentosos e cirúrgicos, conforme delineado por Engelhorn..$^{13}$ Para definir o grupo de risco para trombose venosa profunda foi utilizada a classificação recomendada pela Sociedade Brasileira de Angiologia e Cirurgia Vascular. $^{6}$

O estudo foi condicionado ao cumprimento dos princípios éticos contidos na Declaração de Helsinki da World Medical Association e foi regido por anuência formal da Instituição para a divulgação dos dados levantados. A análise percentual dos dados levantados foi realizada de acordo com a freqüência e de adesão ao programa de profilaxia da trombose venosa profunda, calculada para cada grupo de risco desta patologia.

\section{Resultados}

O gráfico 1 representa o total de pacientes monitorados no período de agosto a novembro de 2003 no Instituto Nacional de Traumato-Ortopedia/RJ. O gráfico 2 demonstra a estratificação das cirurgias ortopédicas realizadas no período de agosto a novembro de 2003. No gráfico 3, os dados referentes à idade dos pacientes monitorados demonstram a maior concentração de indivíduos entre 61 e 70 anos, fato este relevante para a avaliação de fatores de risco para TVP. O gráfico 4 demonstra o número de pacientes monitorados que apresentaram fatores de risco para TVP no período estudado. O gráfico 5 aponta dados referentes aos sintomas sugestivos de TVP e sangramento, que devem ser levados em consideração para o correto diagnóstico e tratamento destes distúrbios.

\section{Discussão}

No final da década de 1960, a história natural do tromboembolismo venoso foi melhor elucidada com contribuições de Kakkar. ${ }^{23}$ Seus estudos demonstraram que o exame clínico da trombose venosa isoladamente é pouco confiável, e métodos como o teste do fibrinogênio marcado e a flebografia apontam que $50 \%$ ou mais dos casos de trombose venosa confirmada não apresentavam sinais clínicos. A partir dessa descoberta, a profilaxia da trombose venosa profunda e do embolismo pulmonar ganhou novos rumos, permitindo que se elaborassem consensos e recomendações para cada grupo de risco dentro dessa entidade. ${ }^{20-22}$ Entretanto, o tromboembolismo venoso continua sendo a maior causa de morte súbita em leitos hospitalares e o principal responsável pela morte de mulheres durante a gestação e o puerpério. ${ }^{10,16}$ Provavelmente, isso ocorre devido à desinformação a respeito da sua incidência, o que leva muitos médicos a subestimá-la. ${ }^{13}$

A atenção farmacêutica realizada com o paciente após a alta hospitalar, em seu domicílio, é de grande relevância na profilaxia da TVP, pois nos últimos anos, com a preocupação sobre custos de internação, as altas hospitalares são cada vez mais precoces, fazendo com que muitos casos de tromboembolismo ocorram no domicilio, após a alta hospitalar. ${ }^{21,22}$ Estudos em pacientes cirúrgicos ortopédicos de alto risco para tromboembolismo venoso revelam que a profilaxia nesses casos deve continuar até cerca de quatro semanas após o procedimento cirúrgico. ${ }^{22}$ Em casos cirúrgicos gerais, até $25 \%$ dos casos de tromboembolismo podem ocorrer no ambiente domiciliar, após a alta. A monitoração recomendada chega a um total de oito semanas, o dobro do preconizado, o que permite uma abordagem maior junto ao paciente, diminuindo dessa forma as chances do desencadeamento da TVP. ${ }^{21,22}$

Em nosso estudo foram monitorados após a alta hospitalar 113 pacientes, sendo 46 (51\%) homens e 67 (59\%) mulheres, conforme mostra o gráfico 1 . Observa-se um número maior de mulheres monitoradas; estas, muitas vezes,

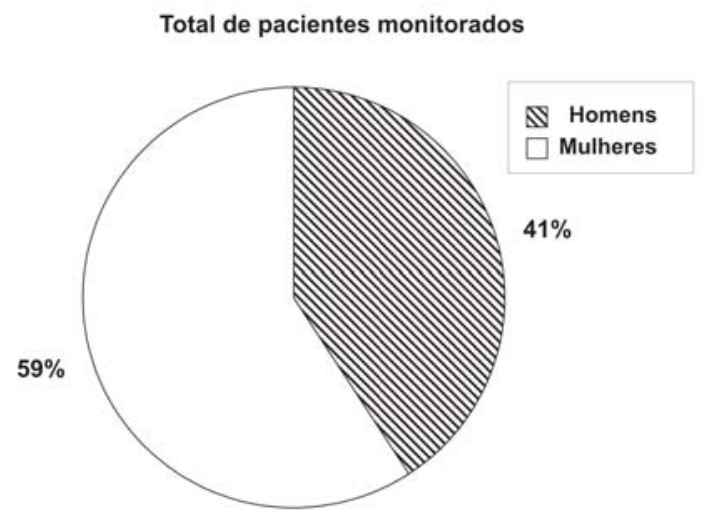

Gráfico 1 
por fazerem uso de anticoncepcionais apresentam maior propensão ao desenvolvimento de varizes (para cada um homem com varizes, há três mulheres), e tornam-se mais suscetíveis ao desenvolvimento de TVP; tais fatores de risco, somados à cirurgia ortopédica, devem ser considerados na prevenção da TVP. Caso ocorra desenvolvimento de varizes, o farmacêutico deve orientar o paciente a fazer uso do tratamento com meias elásticas, que têm a finalidade de comprimir as zonas afetadas pelas varizes, favorecendo assim a passagem do sangue venoso das extremidades inferiores às veias, que o devolvem ao coração. Quanto ao uso de anticoncepcional, deve-se orientar a paciente a conversar com o médico, para que se avalie a real necessidade do uso. ${ }^{3}$

O gráfico 2 mostra que um alto número de pacientes realizou cirurgia de quadril -75 (66,37\%); este tipo de cirurgia é considerada de grande porte. Analisando-se o gráfico 3, observa-se que a idade dos pacientes, em sua grande maioria, está acima dos 40 anos $(90,27 \%)$, constituindo desta forma fator de risco para desenvolvimento da TVP. Estes dados denotam a importância da atenção farmacêutica na profilaxia da TVP em pós-operatório pois, sem esta, um em cada quatro pacientes com idade superior a 40 anos, submetidos à cirurgia de grande porte, com mais de uma hora de duração, desenvolve TVP. ${ }^{32,33}$ Tal situação enquadra quase na totalidade os pacientes monitorados nesse estudo.

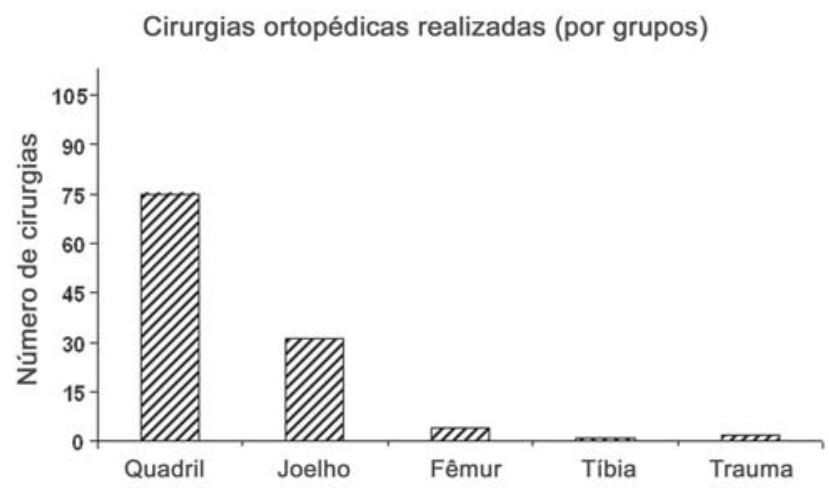

Gráfico 2

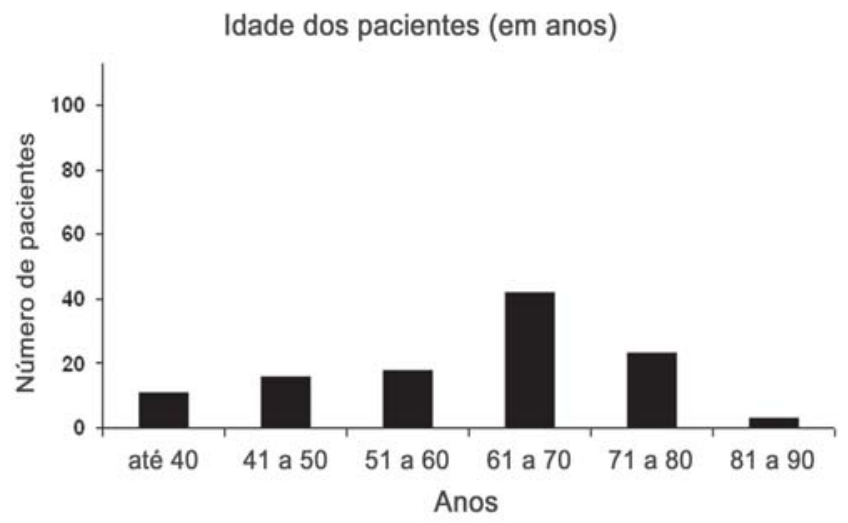

Gráfico 3
Analisando-se o gráfico 4, verifica-se a presença de fatores de risco para TVP nos pacientes; esses fatores são condições que favorecem a instalação desta patologia. É importante ressaltar que os pacientes monitorados neste estudo, por terem realizado cirurgias ortopédicas, são considerados pacientes de alto risco para desenvolvimento da TVP. ${ }^{6} \mathrm{~A}$ concomitância desses eventos (fatores de risco mais cirurgia ortopédica) tem efeito sinérgico com o aumento de risco para TVP, o que torna esses pacientes merecedores de atenção e cuidados especiais. ${ }^{12,36} \mathrm{O}$ farmacêutico deve estimular o paciente a aderir ao tratamento, que inclui medidas profiláticas físicas e medicamentosas, além de orientá-lo a respeito da patologia.

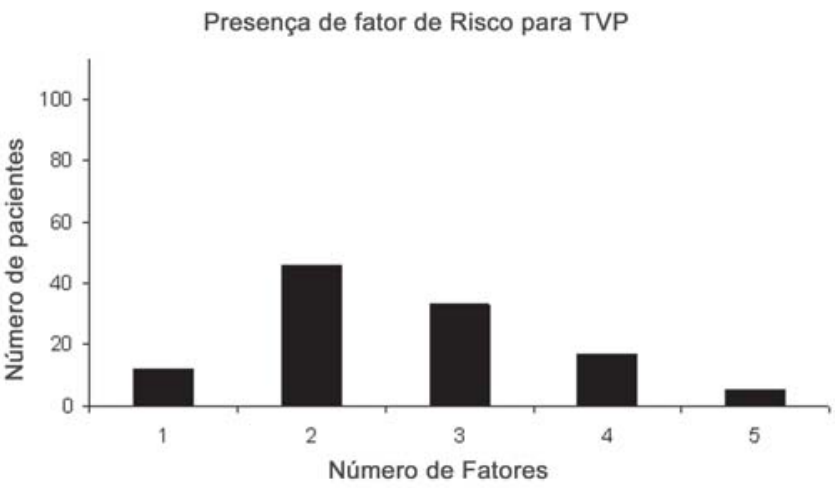

Gráfico 4

O gráfico 5 mostra que 37 (32,74\%) pacientes apresentaram sintomas sugestivos da TVP. A identificação desses sintomas constitui a grande importância deste trabalho de atenção farmacêutica, pois a literatura fornece o embasamento necessário que aponta que tais manifestações clínicas podem denotar o início de um quadro de TVP. ${ }^{27}$ Desta forma, a atenção farmacêutica torna-se uma ferramenta de grande importância na prevenção desta patologia, pois, ao ser diagnosticada precocemente, a cura torna-se mais fácil, não desencadeando ao paciente comprometimento na sua qualidade de vida (o que poderia ocorrer caso seja diagnosticada tardiamente).

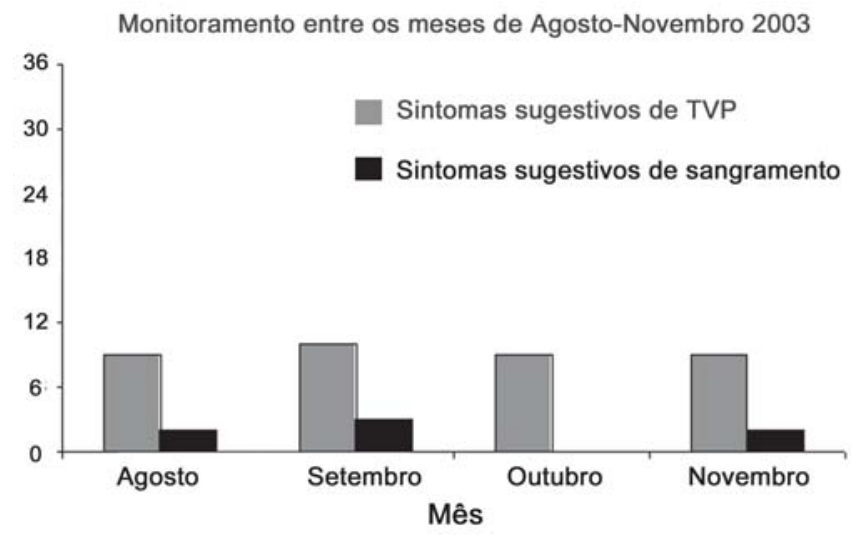

Gráfico 5 
Sintomas sugestivos de sangramento foram apresentados por sete $(6,19 \%)$ pacientes, e estes dados confirmam relatos da literatura, visto que o uso de heparina de baixo peso molecular diminui a propensão a hemorragias. ${ }^{26,32}$ Acredita-se também que este episódio deve-se à prescrição de antiinflamatórios não estereoidais (AINEs) por muitos médicos na alta hospitalar, embora o paciente seja orientado pelo farmacêutico a não fazer uso concomitante com a heparina de baixo peso molecular; entretanto, muitos o fazem e, desta forma, potencializam o desencadeamento de hemorragias, pois estes (enoxaparina e aintiinflamatórios) possuem atividade antitrombótica . $^{17,24}$

A título de informação adicional, é válido destacar que a monitoração não foi realizada em nove pacientes, devido à dificuldade de comunicação via telefone. Dois pacientes não aderiram ao tratamento: um internou-se em outro hospital - porém foi realizada a monitoração - e o outro saiu do hospital com urticárias, cujo quadro ficou mais grave após a alta. Um paciente desenvolveu trombos e embolia pulmonar, sendo internado em outro hospital. Este ocorrido denota que a profilaxia medicamentosa para tromboembolismo venoso, apesar de efetiva, não reduz a zero a incidência de TVP pós-operatória. Dados da literatura indicam a incidência de TVP pós-operatória nos casos de cirurgia de quadril; se nada for feito em termos de profilaxia, até $70 \%$ dos pacientes podem desenvolver tromboembolismo venoso. ${ }^{10}$ Caso seja utilizada heparina não fracionada, esta incidência cai para cerca de $25 \%$, e para $12 \%$ se for utilizada heparina de baixo peso molecular. Verifica-se através destes dados que as incidências ainda são consideráveis, o que torna de grande relevância a atenção farmacêutica realizada no pós-operatório, com a finalidade de prevenir esta terrível patologia. ${ }^{5}$

Todos os pacientes monitorados receberam profilaxia. Deve-se levar em consideração que o estudo envolveu uma amostra de pacientes originada de um único hospital, de forma que não deve ser generalizada para os demais hospitais, pois verifica-se que a profilaxia da TVP, embora tenha sua eficácia comprovada por diversos estudos, não é praticada por muitos profissionais da área de saúde.

Um estudo realizado no Hospital de Clínicas de Porto Alegre demonstrou que a profilaxia medicamentosa para a TVP é empregada em apenas 50\% dos pacientes com indicação para utilizá-la, mesmo em hospitais universitários. ${ }^{28}$ Ademais, um levantamento feito por $\mathrm{Maffei}^{27} \mathrm{com}$ trinta médicos no Brasil, revelou que apenas 15,6\% tinham pleno conhecimento da incidência do tromboembolismo venoso. Outro estudo realizado nos Estados Unidos com mais de 2 mil pacientes internados em 16 hospitais mostrou que apenas um terço dos pacientes recebeu profilaxia apesar de apresentarem vários fatores de risco para TVP. ${ }^{2}$

Guillies e colaboradores ${ }^{19}$ analisaram casos de embolia pulmonar fatal durante um ano e concluíram que 56\% dos pacientes não receberam profilaxia, apesar de apresentarem fatores de risco para TVP e nenhuma contra-indicação para o uso de anticoagulantes. Uma possível explicação para a não-utilização da profilaxia para a trombose venosa em pacientes cirúrgicos é a preocupação dos cirurgiões quanto ao risco de sangramento durante a operação que, teoricamente, o uso de anticoagulante pode acarretar. Contudo, dados de metanálises e estudos randomizados duplo-cegos com placebo demonstraram que não há aumento significativo de sangramento com uso da heparina não fracionada em baixa dose e, principalmente, de heparina de baixo peso molecular. ${ }^{31,32}$ Outra explicação para a não utilização da profilaxia é o custo financeiro que ela pode acarretar. Porém, Bergqvist e colaboradores ${ }^{4}$ e Gerhardt ${ }^{16}$ afirmam que a utilização da profilaxia, quando indicada corretamente, apresenta custo-benefício positiva.

O farmacêutico pode contribuir para a realização da profilaxia da TVP através de programas educacionais sobre o assunto para profissionais da área da saúde, sendo esta prática de extrema importância. Um estudo prospectivo de Anderson e colaboradores ${ }^{2}$ documentou um aumento no uso da profilaxia de $29 \%$ para $52 \%$ em pacientes hospitalizados com risco potencial para desenvolver trombose venosa, após a instituição de estratégias educacionais com o propósito de alertar os profissionais para a importância da prevalência do tromboembolismo.

A utilização da profilaxia foi superior nos hospitais nos quais médicos participavam continuamente dos programas de educação; um fator chave que o farmacêutico pode utilizar nos programas educacionais para motivar a realização da profilaxia é trazer ao conhecimento dos profissionais de saúde as estatísticas da doença tromboembólica no hospital onde trabalham.

\section{Conclusões}

O farmacêutico, inserido no contexto da multidisciplinaridade, colocando em prática a atenção farmacêutica, torna-se uma peça importante tanto na profilaxia da TVP e tratamento dos problemas relacionados ao medicamento, quanto dos problemas de saúde, complementando a eficácia da recuperação da saúde do paciente, contribuindo para melhora da sua qualidade de vida. Este estudo não permite a identificação da prevalência de TVP e suas complicações, pois muitos pacientes que desenvolvem esta patologia não são tratados no Instituto Nacional de Traumato-Ortopedia (Into/RJ), em virtude deste hospital ser de especialidade ortopédica.

Tais pacientes são encaminhados para hospitais que possuem clínicas de especialidade vascular. Todavia, foi possível detectar o alto índice de TVP no período de agosto a novembro de 2003, fornecido por estudos realizados por cirurgiões, o que revela sua importância relacionada à pratica de atenção farmacêutica e seu papel fundamental na prevenção desta doença. 


\begin{abstract}
Venous thromboembolism is a potentially fatal pathology that can complicate the recovery of hospitalized patients. Surgery patients, especially those submitted to major surgical procedures, are particularly at risk. Deep venous thrombosis (DUT) and pulmonary embolism are recognized postoperative complications whose diagnosis is difficult and whose treatment is frequently initiated too late to be effective. Without prophylaxis one in every four over 40year-old patients who are submitted to general surgery of more than one hour will develop DVT. At-risk patients can be identified and receive prophylaxis - the least onerous method to reduce morbidity and mortality. Thus, the practice of Pharmaceutical care, use in the postoperative period of orthopedic surgery, is a useful tool to avoid the progression of this pathology. Through the identification of suggestive symptoms, it is possible to prevent the aggravation of this disease, facilitating its treatment. Rev. bras. hematol. hemoter. 2008;30(1):29-35.
\end{abstract}

Key words: Deep venous thrombosis; pharmaceutical care; low weight heparin.

\section{Agradecimentos}

Agradecemos à Dra. Elisabete Regina Viana/ Instituto Nacional de Traumato-Ortopedia/Ministério da Saúde pelo apoio técnico e científico.

\section{Referências Bibliográficas}

1. Albuquerque LC et al. Terapia trombolítica em trombose venosa profunda. Experiência clínica inicial. Arq Bras Cardiol. 1997: 68:1-6.

2. Anderson FA Jr et al. A population based perspective of the hospital incidence and case fatality rates of deep vein thrombosis and pulmonary embolism. Arch Intern Med. 1991;151:933-8.

3. Baruzzi ACA et al. Trombose venosa profunda. Profilaxia. Arq Bras Cardiol. 1996;67:1-8.

4. Bergqvist D. Prevention of postoperative thromboembolism in Sweden: the development of practice during five years. Thromb Haem. 1985;56:239-41.

5. Boneu B. Low molecular weight heparin therapy: is monitoring needed? Thromb Haemost. 1994;72:330-4.

6. Caiafa JS. Medidas profiláticas da doença tromboembólica. In: Thomas J.B. Síndromes venosas: diagnóstico e tratamento. Rio de Janeiro: Revinter, 2001.

7. Castro Silva M. Epidemiologia do tromboembolismo venoso. J Vasc Br. 2002:1:83-4.

8. Castro Silva M. Tromboembolismo venoso - epidemiologia e fatores de risco. In: Brito CJ et al. (eds.). Cirurgia Vascular. Rio de Janeiro: Editora Revinter; 2002. p. 1123-34.

9. Castro Silva M. Venous thromboembolism in the State of Minas Gerais and its projection to Brazil: study based in 2,331,353 hospitalizations. Intern Angiol. 1997;16:193-6.

10. Clagett GP et al. Prevention of venous thromboembolism. Chest. 1998;114:531S-560S.

11. Dupim JAA. Assistência Farmacêutica. Um modelo de organização. Belo Horizonte, 1999.

12. Duque FLV, Mello NA. Trombogênese - Trombofilia. J. Vasc. Br. 2003;2:105-18.
13. Engelhorn ALV et al. Profilaxia da trombose venosa profunda - estudo epidemiológico em um hospital escola. J Vasc Br. 2002;1:97-102.

14. Geerts WH et al. Prevention of venous thromboembolism. Chest. 2001;119:132S-75S.

15. Gerez JC. Indústria farmacêutica: Histórico, mercado e competição. Ciência Hoje. 1993;15:21-30.

16. Gerhardt A, Scharf RE, Beckmann MW et al. Prothrombin and factor $\mathrm{V}$ mutations in women with history of thrombosis during pregnancy and the puerperium. N Engl J Med. 2000;342:374-80.

17. Goodman L, Gilman A. As Bases Farmacológicas da Terapêutica. $9^{\mathrm{a}}$ ed. Rio de Janeiro: Mc Graw-Hill, 1996.

18. Gouveia WA. At center stage: Pharmacy in the next century. Am J Health Syst Pharm. 1999;56:1-11.

19. Guilles TE et al. Still missing the boat with fatal pulmonary embolism. Br J Surg. 1996;83:1394-5.

20. Hepler CD, Strand LM. Opportunities and responsabilities in pharmaceutical care. Am J Hosp Pharm. 1990;47:533-43.

21. Huber $\mathrm{O}$ et al. Postoperative pulmonary embolism after hospital discharge. An underes limaled risk. Arch Surg. 1992;127:310.

22. Hull RD, Pineo GF. Prophylaxis of deep venous thrombosis and pulmonary embolism. Current recommendations. Med clin North Am. 1998;82:477-93.

23. Kakkar VV et al. Natural history of postoperative deep vein thrombosis. Lancet. 1969;2:230-2.

24. Katzung BG. Farmacologia Básica e Clínica. Rio de Janeiro: Guanabara Koogan, 2003.

25. Lastoria S. Trombose venosa profunda em coto de amputação: estudo pelo mapeamento dúplex. Cir Vasc Angiol. 1995;11:27.

26. Maciel R. Heparina de baixo peso molecular no tratamento da tromboembolia pulmonar. J Pneumol. 2002;28:137-42.

27. Maffei FHA. Profilaxia da trombose venosa e da embolia pulmonar. In Maffei FHA et al. Doenças vasculares periféricas. $3^{\text {a }}$ ed. São Paulo: Medsi, 2002.

28. Menna-Barreto SS et al. Estratificação de risco e profilaxia para tromboembolia venosa em pacientes internados em hospital geral universitário. J Pneumol. 1998;24:298-302.

29. Nau DP et al. Preparing pharmacists for pharmaceutical care. J Am Pharm Assoc. 1998;38:644.

30. Nicolaides AN. Prevention of venous thromboembolism. Int Ang. 1997;16:3-38.

31. Nurmohamed MT et al. Low molecular weight heparin versus standard heparin in general and orthopaedic surgery: a meta analysis. Lancet. 1992;340:152-5.

32. Rhodia Farma. Clexane Enoxaparina. Monografia Científica. s/a, 1995.

33. Roberts HR. Prevention of venous thrombosis and pulmonary embolism. JAMA. 1986;256:744-9.

34. Thompson CA. Restructuring and patient-focuse care (editorial). Am J Hosp Pharm. 1995;52:41.

35. Warkentin TE. Heparin-induced thrombocytopenia in patients treated with low-molecular-weigth heparin or unfractionated heparin. N Engl J Med. 1995;332:1330-5.

36. Weinmann EE, Salzman E. Deep-vein thrombosis. N Engl J Med. 1994;331:1630-40.

Avaliação: Editor e dois revisores externos

Conflito de interesse: sem conflito de interesses

Recebido: 30/10/2006

Aceito após modificações: 07/08/2007 